This document is confidential and is proprietary to the American Chemical Society and its authors. Do not copy or disclose without written permission. If you have received this item in error, notify the sender and delete all copies.

\title{
Interaction of Azole-based Environmental Pollutants with the Coelomic Hemoglobin from Amphitrite ornata: a Molecular Basis for Toxicity
}

\begin{tabular}{|r|l|}
\hline Journal: & Biochemistry \\
\hline Manuscript ID & bi-2017-00041d.R1 \\
\hline Manuscript Type: & Article \\
\hline Date Submitted by the Author: & O6-Apr-2017 \\
\hline Complete List of Authors: & $\begin{array}{l}\text { McCombs, Nikolette; North Carolina State University, Department of } \\
\text { Chemistry } \\
\text { Moreno Chicano, Tadeo; University of Essex, School of Biological Sciences } \\
\text { Carey, Leiah; North Carolina State University, Department of Chemistry } \\
\text { Franzen, Stefan; NC State University, Chemistry } \\
\text { Hough, Michael; University of Essex, School of Biological Sciences } \\
\text { Ghiladi, Reza; North Carolina State University, Department of Chemistry }\end{array}$ \\
\hline
\end{tabular}

\section{SCHOLARONE \\ Manuscripts}




\title{
Interaction of Azole-based Environmental Pollutants
}

\author{
with the Coelomic Hemoglobin from Amphitrite
}

ornata: a Molecular Basis for Toxicity

Nikolette L. McCombs ${ }^{1}$, Tadeo Moreno-Chicano ${ }^{2}$, Leiah M. Carey ${ }^{1}$, Stefan Franzen ${ }^{1}$, Michael A.

Hough $^{2, *}$ and Reza A. Ghiladi ${ }^{1, *}$

${ }^{1}$ Department of Chemistry, North Carolina State University, Raleigh, North Carolina, 27695-

8204

${ }^{2}$ School of Biological Sciences, University of Essex, Wivenhoe Park, Colchester, Essex, CO4

3SQ, UK

Manuscript Correspondence:

Prof. Reza A. Ghiladi

Department of Chemistry

North Carolina State University

Raleigh, NC 27695

(919) 513-0680 (phone)

(919) 515-8920 (fax)

E-mail: Reza_Ghiladi@ncsu.edu

Dr. Michael A. Hough

School of Biological Sciences

University of Essex

Wivenhoe Park, Colchester

CO4 3SQ, Essex, UK

Tel: +44 1206873317

mahough@essex.ac.uk 


\begin{abstract}
Abbreviations
BIm, benzimidazole; Bta, benzotriazole; Compound I, two-electron oxidized heme center when compared to the ferric form, commonly as an $\mathrm{Fe}^{\mathrm{IV}}=\mathrm{O}$ porphyrin $\pi$-cation radical; Compound II, one-electron oxidized heme center when compared to the ferric form, commonly as an $\mathrm{Fe}^{\mathrm{IV}}=\mathrm{O}$ or $\mathrm{Fe}^{\mathrm{IV}}-\mathrm{OH}$; Compound ES, two-electron-oxidized state containing both a ferryl center $\left[\mathrm{Fe}^{\mathrm{IV}}=\mathrm{O}\right]$ and an amino acid (tryptophanyl or tyrosyl) radical, analogous to Compound ES in cytochrome c peroxidase; Compound RH, 'Reversible Heme' state of dehaloperoxidase, formed from the decay of Compound ES in the absence of co-substrate; CT1, charge transfer 1; DFT, density functional theory; DHP, dehaloperoxidase; hhMb, horse heart myoglobin; HS, high-spin; Im, imidazole; Inz, indazole; LS, low-spin; POP, persistent organic pollutant; SCRR, single crystal resonance Raman; TBP, 2,4,6-tribromophenol; TCP, 2,4,6-trichlorophenol; WT, wild-type; 4BP, 4-bromophenol; 5c, 5-coordinate; 6c, 6-coordinate.
\end{abstract}




\begin{abstract}
The toxicities of azole pollutants that have widespread agricultural and industrial uses are either poorly understood or unknown, particularly with respect to how infaunal organisms are impacted by this class of persistent organic pollutant. To identify a molecular basis by which azole compounds may have unforeseen toxicity on marine annelids, we examine here their impact on the multifunctional dehaloperoxidase (DHP) hemoglobin from the terebellid polychaete Amphitrite ornata. UV-visible and resonance Raman spectroscopic studies showed an increase in the 6-coordinate low spin heme population in DHP isoenzyme B upon binding of imidazole, benzotriazole, and benzimidazole $\left(K_{\mathrm{d}}\right.$ values $=52,82$, and $110 \mu \mathrm{M}$, respectively), suggestive of their direct binding to the heme-Fe. Accordingly, atomic resolution X-ray crystal structures, supported by computational studies, of the DHP B complexes of benzotriazole (1.14 $\AA)$, benzimidazole $(1.08 \AA)$, imidazole $(1.06 \AA)$, and indazole $(1.12 \AA)$ revealed two ligand binding motifs, one with direct ligand binding to the heme-Fe, and another where the ligand binds in the hydrophobic distal pocket without coordinating the heme-Fe. Taken together, the results demonstrate a new mechanism by which azole pollutants can potentially disrupt hemoglobin function, thereby increasing our understanding of their impact on infaunal organisms in marine and aquatic environments.
\end{abstract}


A number of persistent organic pollutants (POPs) are comprised of five-membered nitrogen (N)-containing heterocyclic rings belonging to the azole class of compounds. Such compounds, examples of which include benzotriazole (Bta), benzimidazole (BIm), indazole (Inz), and imidazole (Im), as well at their derivatives, have found a number of industrial and agricultural uses. Benzotriazoles have been used as UV absorbers to protect polymers from photochemical degradation, and more recently as anticorrosive additives (e.g., in cooling and hydraulic fluids, antifreeze products, dishwasher detergents). ${ }^{1,2}$ Recalcitrant to wastewater treatment where effluent concentrations are as high as $10 \mu \mathrm{M},{ }^{1,3,4}$ they have been detected in rivers, lakes and groundwater with concentrations as high as $1032 \mathrm{ng} / \mathrm{L}$ for benzotriazole and $516 \mathrm{ng} / \mathrm{L}$ for methylbenzotriazole, and have been reported as the fourth most common European groundwater pollutant. ${ }^{5}$ Likewise, benzimidazole and its derivatives have been used both as fungicides for crop protection (applied directly to soil or sprayed over fields), ${ }^{6-8}$ and as veterinary antiparasitic drugs administered to livestock (cows, sheep, hogs), ${ }^{9,}{ }^{10}$ where they have been shown to enter into the environment primarily through excretion (urine or feces). Similarly, indazole ${ }^{11}$ and imidazole ${ }^{12-14}$ derivatives have a wide variety of industrial, pharmaceutical, and agricultural uses. Importantly, no azole-containing compounds are on the EPA priority pollutant list, and as such these and similar compounds are often overlooked as environmental contaminants.

POPs enter marine and freshwater ecosystems through effluent releases, atmospheric deposition, runoff, and other means, where their low water solubility results in strong binding to particulate matter in aquatic sediments. ${ }^{15}$ As a result, sediments can serve as reservoirs or "sinks" for azole POPs that lead to their exposure to sediment-dwelling organisms, ${ }^{9}$ either through contact upon burrowing or via ingestion by deposit feeders. With very limited data available, their persistence and fate in marine environments, as well as their toxicities to marine organisms, 
are either unknown or poorly understood, with an added caveat that studies have shown that some azole metabolites are more toxic than the parent compound. ${ }^{7,}{ }^{16}$ Thus, as relatively little is known regarding the underlying molecular basis for how infaunal systems are impacted by the azole class of POPs, this lack of knowledge may lead to i) an underestimation of the environmental toxicity of azole compounds that are currently in production or use, ii) approval of other azole compounds for industrial or agricultural use despite their unknown potential for harming marine environments, iii) limited cleanup or (bio)remediation efforts upon environmental contamination due to a lack of concern regarding such compounds, and iv) a lack of awareness of the individual or combined effects of POPs and their metabolites, particularly with respect to their bioaccumulation, transport, and fate through environments where marine annelid exposure is substantial. Thus, given their growing use across a breadth of applications, it is of increasing importance to achieve a full understanding of how azole POPs and their metabolites may impact marine environments.

Our platform for assessing the molecular basis by which azole compounds may have unforeseen effects on infaunal organisms is the coelomic hemoglobin from the terebellid polychaete Amphitrite ornata. ${ }^{17}$ In addition to its role in oxygen transport, ${ }^{17}$ this multifunctional hemoprotein, historically named dehaloperoxidase (DHP) due to its ability to catalyze the oxidative dehalogenation of 2,4,6-trihalophenols to their corresponding 2,6 -dihaloquinones, ${ }^{18-21}$ is believed to have evolved in A. ornata to detoxify high levels of volatile organobromine secondary metabolites secreted as repellents against predation by neighboring sediment-dwellers in benthic ecosystems. In addition to this biologically-relevant peroxidase activity, the two isoenzymes of DHP (A and $\mathrm{B})^{22}$ have recently been shown to possess peroxygenase activity against both native haloindole ${ }^{23}$ and anthropogenic nitrophenol ${ }^{24}$ substrates. As such, A. ornata 
DHP represents a multifunctional catalytic globin that possesses a broad substrate scope with activities commonly associated with the peroxidase and P450 families of enzymes. ${ }^{25}$ Thus, given its native peroxygenase activity against naturally-occurring halogenated indoles that are structurally related to the aforementioned azole pollutants, questions thus arise as to whether DHP binds, and/or potentially reacts with, similar compounds of anthropogenic origin, the answers to which may potentially provide insight into previously unknown molecular mechanisms for how infaunal organisms may be impacted by POPs.

To address these questions, here we have explored heterocyclic azole compounds, comprising benzotriazole, benzimidazole, indazole and imidazole (Figure 1), for their ability to bind to and possibly modulate the function of DHP. Using a combination of substrate binding studies, biochemical assays, solution and crystal resonance Raman spectroscopy, and X-ray crystallography, we have identified a previously unknown binding motif for azole compounds with the hemoglobin of $A$. ornata. More importantly, however, the results demonstrate that there may be a much larger number of heterocyclic compounds of anthropogenic origin that can bind to marine hemoglobins, leading to a new mechanism by which pollutants can disrupt hemoglobin function, a finding which may be important when assessing the environmental impact of chemicals such as POPs and their metabolites on aquatic systems.<smiles>c1ccc2[nH]cnc2c1</smiles><smiles>CCOCCO</smiles><smiles>c1ccc2[nH]nnc2c1</smiles><smiles>c1ccc2[nH]ncc2c1</smiles>

Benzotriazole (Bta) Indazole (Inz)

Figure 1. Azole ligands investigated for their binding to DHP. 


\section{EXPERIMENTAL}

Materials and Methods. Reagent-grade chemicals (VWR, Sigma-Aldrich or Fisher Scientific) were used without further purification. Acetonitrile was HPLC grade. UV-visible spectroscopy was performed aerobically on a Varian Cary Bio50 spectrophotometer, or anaerobically on an Agilent 8454 diode-array spectrophotometer in a glovebox. Stock solutions (10-50 mM) of all compounds were prepared in $\mathrm{MeOH}$, stored at $4{ }^{\circ} \mathrm{C}$, and were periodically screened by HPLC to ensure that they had not degraded. Aliquots were stored on ice during use. Solutions of $\mathrm{H}_{2} \mathrm{O}_{2}$ were prepared fresh daily and kept on ice until needed. $\mathrm{H}_{2} \mathrm{O}_{2}$ concentration was determined by UV-vis $\left(\varepsilon_{240}=46 \mathrm{M}^{-1} \mathrm{~cm}^{-1}\right){ }^{26} \mathrm{DHP}$ variants were expressed and purified as previously reported. $^{27-29}$ Oxyferrous DHP was obtained by the aerobic addition of excess ascorbic acid to a solution of ferric DHP, followed by desalting (PD-10 column). Enzyme concentration was determined spectrophotometrically using $\varepsilon_{\text {Soret }}=116,400 \mathrm{M}^{-1} \mathrm{~cm}^{-1}$ for all isoenzymes. ${ }^{28}$ Anaerobic studies were performed in an MBraun Labmaster 130 nitrogen-filled glovebox using Ar-degassed solutions of buffer, peroxide, substrate and enzyme.

Substrate-Binding Studies. Adapted from previously published protocols, ${ }^{30} 50 \mathrm{mM}$ azole stock solutions were prepared in $100 \mathrm{mM} \mathrm{KP}_{\mathrm{i}}$ buffer $(\mathrm{pH}$ 7). The UV-visible spectrophotometer was first blanked using a solution of $10 \mu \mathrm{M}$ ferric wild-type (WT) DHP B in buffer containing $10 \% \mathrm{MeOH}$. Difference spectra were then acquired in the presence of 2.5-100 eq. azole while maintaining both constant enzyme and $\mathrm{MeOH}$ concentrations. Analysis by nonlinear regression $^{23,24}$ using the GraFit software package (Erithacus Software Ltd.) of the experiments performed in triplicate provided a calculated $\mathrm{A}_{\max }$, which was in turn used to calculate $\alpha$ for the average $\Delta \mathrm{A}$ for each substrate concentration. A nonlinear regression plot provided the reported 


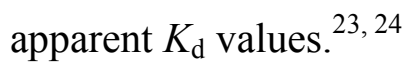

Resonance Raman Studies. Samples for solution resonance Raman spectroscopic studies were prepared with final concentrations of $75 \mu \mathrm{M}$ WT DHP B and $3.75 \mathrm{mM}$ azole in $100 \mathrm{mM}$ $\mathrm{KP}_{\mathrm{i}}(\mathrm{pH} 7$ or 9) containing $10 \% \mathrm{MeOH}(\mathrm{v} / \mathrm{v})$, and then transferred to a 5-mm o.d. glass NMR tube. Anaerobic samples were prepared inside a glovebox in the same fashion using septum capped NMR tubes. Spectra were obtained by Soret band excitation $(400-430 \mathrm{~nm} ; 60 \mathrm{~mW}$ power) as previously described. ${ }^{23}$

In order to validate the ligand states in DHP-azole crystals, additional single crystal resonance Raman data (SCRR) were measured, from separate crystals, using the on-line MS3 microspectrophotometer $^{31}$ at the Swiss Light Source beamline X10SA with an excitation wavelength of $405 \mathrm{~nm}$. Raman shifts were calibrated using 4-acetaminophen as reference, and all data were analysed using the SLS-APE ${ }^{32}$ in-house suite. Several RR spectra were measured from each crystal prior to X-ray exposure in order to test for laser-induced photoreduction of the heme-Fe. Averaged, accumulated spectra were measured for each DHP-azole crystal prior to, and following, measurement of X-ray data (diffraction data not shown).

Protein Crystallization and X-ray Diffraction Studies. Crystals of non His-tagged recombinant DHP $\mathrm{B}^{24}$ were obtained through the hanging-drop vapor diffusion method: 12 $\mathrm{mg} / \mathrm{mL}$ DHP $\mathrm{B}$ in $20 \mathrm{mM} \mathrm{Na}$ cacodylate ( $\mathrm{pH}$ 6.4) was mixed with a reservoir solution comprising 14-20\% (w/v) PEG 4000 and 150-250 mM ammonium sulfate. Drops were prepared using 1:1 and 1:2 protein:reservoir ratios, obtaining final drop volumes of 4 and $6 \mu \mathrm{L}$, respectively. Crystals grew at $4{ }^{\circ} \mathrm{C}$ in 3-6 days. DHP B ferric crystals were soaked in reservoir solutions supplemented with either imidazole $(5 \mathrm{mM})$ or indazole, benzimidazole or benzotriazole (10 mM, each in 2\% DMSO). Crystals were soaked for 5-10 min, before transfer to 
a cryo-solution containing reservoir solution supplemented with $20 \%$ glycerol prior to flashfreezing in liquid nitrogen.

Data were measured to atomic resolution for DHP B ligand complexes using Diamond Light Source beamline I04-1. Data measured at Diamond were processed automatically in Xia2 using $\mathrm{XDS}^{33}$ and Aimless ${ }^{34}$ with the resolution limit based on a criterion of $\mathrm{CC}^{1 / 2}>0.5$ for the highest resolution shell. Data from SLS were manually processed using XDS and Aimless using the same criteria. Structures were refined by maximum likelihood methods in Refmac5 within the CCP4 suite using the CCP4i2 GUI. Ligands were modelled into strong sigmaA-weighted Fo-Fc electron density features with ligand chemical structures and restraints taken from the HIC-UP ${ }^{35}$ database (Uppsala) and from SMILES. Validation of structures utilised MolProbity ${ }^{36}$, the JCSG Quality Control Server and the PDB validation server. Coordinates and structure factors were deposited in the RCSB Protein Data Bank with accession codes given in Table S1. Absorbed Xray doses were determined using Raddose $3 \mathrm{D} .{ }^{37}$

Computational Methodology. All calculations were performed using the Gaussian 09 software package. Structures were optimized in the low spin doublet state in vacuum utilizing the B3LYP functional and 6-31G* basis set for $\mathrm{C}, \mathrm{N}, \mathrm{O}, \mathrm{H}$ and the double- $\zeta$ basis set of Schafer et al., ${ }^{38}$ enhanced with an extra $\mathrm{p}$ function (exponent 0.134915 ) on Fe. The geometry optimized structures were carried out until reaching an energy difference of $10^{-8}$ Ha on successive iterations without constraints. A protoporphyrin IX model was employed that replaced the propionate arms with methyl groups, and the proximal histidine with a methylimidazole axial ligand. 


\section{RESULTS}

UV-visible Spectroscopic Studies. The electronic absorption spectra in $100 \mathrm{mM} \mathrm{KP}$ ( $\mathrm{pH} 7$ ) containing $10 \% \mathrm{MeOH}(\mathrm{v} / \mathrm{v})$ at $25{ }^{\circ} \mathrm{C}$ for ferric DHP B alone [407 (Soret), 507, 540 (sh), 635 $\mathrm{nm}$; black] and in the presence of 100 eq. Im [416 (Soret), 537, $565 \mathrm{~nm}$; pink], Bta [418 (Soret), 541, 575 (sh); blue], BIm [410 (Soret), 537, 565 (sh); green], and Inz [408 (Soret), 506, 635 nm; red] are shown in Figure 2A. Relevant spectral features and analysis are found in Table 1, and additional discussion supporting the oxidation state assignment of the DHP-azole adducts as containing 6cLS ferric hemes (as opposed to 6cLS ferrous) can be found in the Supporting Information. 

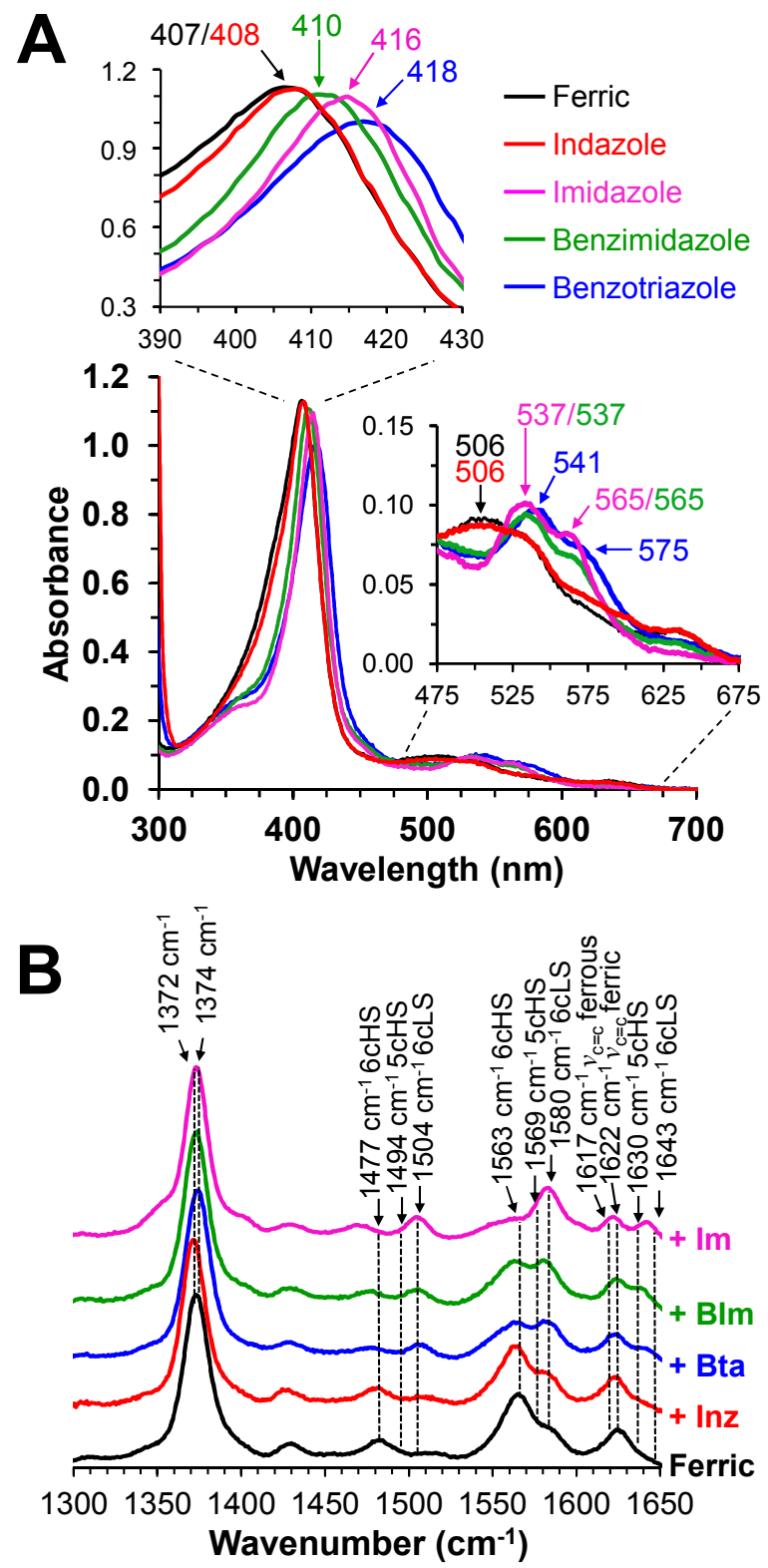

Figure 2. (A) UV-visible spectra of WT ferric DHP $(10 \mu \mathrm{M}$, black $)$ in the presence of 100 equiv. of imidazole (pink), benzimidazole (green), benzotriazole (blue), and indazole (red) in 10\% $\mathrm{MeOH} / 100 \mathrm{mM} \mathrm{KP}$ (v/v) at $\mathrm{pH}$ 7. (B) Resonance Raman spectra of DHP-azole complexes as in panel $\mathrm{A}$ at $75 \mu \mathrm{M}$ enzyme and 50 equiv. azole. 
Table 1. UV-visible spectroscopic data for WT DHP B and its azole adducts. ${ }^{\text {a }}$

\begin{tabular}{lccc} 
& $\lambda_{\max }(\mathrm{nm})$ & $\mathrm{A}_{\text {Soret }} / \mathrm{A}_{380}$ & $\mathrm{~A}_{614} / \mathrm{A}_{645}$ \\
\hline DHP B & 407 (Soret), 507, 540 (sh), 635 & 1.86 & 1.11 \\
+imidazole $(\mathrm{Im})$ & 416 (Soret), 537, 565 & 3.74 & 1.64 \\
+benzotriazole (Bta) & 418 (Soret), 541, 575 (sh) & 3.04 & 1.34 \\
+benzimidazole (BIm) & 410 (Soret), 537, 565 (sh) & 3.05 & 1.16 \\
$\quad$ +indazole (Inz) & 408 (Soret), 506, 635 & 2.08 & 1.08 \\
\hline a $10 \% \mathrm{MeOH}(\mathrm{v} / \mathrm{v})$ in $100 \mathrm{mM} \mathrm{KP}_{\mathrm{i}}, \mathrm{pH} 7,25^{\circ} \mathrm{C}, 100$ equiv. azole. & &
\end{tabular}

The shifts in the relative populations of 5-/6-coordinate (5c/6c) high-/low-spin (HS/LS) heme species in DHP B caused by azole binding can be demonstrated by analysis of the $\mathrm{A}_{\text {Soret }} / \mathrm{A}_{380}$ and $\mathrm{A}_{614} / \mathrm{A}_{645}$ ratios $^{29,39,40}$ : the presence of the azole led to an increase in the $\mathrm{A}_{\text {Soret }} / \mathrm{A}_{380}$ ratio, from 3.04 for DHP-Bta and DHP-BIm, to as high as 3.74 for DHP-Im, versus 1.86 for WT DHP B alone. Similarly, the $\mathrm{A}_{614} / \mathrm{A}_{645}$ ratio was also increased per the trend $\operatorname{Im}>\mathrm{Bta}>\mathrm{BIm}>\operatorname{Inz} \approx$ DHP B. Taken together, for Bta, BIm, and Im, the bathochromic shifts observed upon ligand binding (as revealed by the $\mathrm{A}_{\text {Soret }} / \mathrm{A}_{380}$ ratio) and loss of the charge transfer (CT1) feature (highlighted by the $\mathrm{A}_{614} / \mathrm{A}_{645}$ ratio) were suggestive of a 6cLS heme in DHP. Notably, indazole binding did not significantly alter the spectral features of the starting ferric enzyme. Overall, these UV/vis spectroscopic results for azole binding are in good agreement with the interpretations provided by resonance Raman spectroscopy (vide infra).

Additionally, a pH-dependence on the spectral features consistent with the known alkaline transition $^{41}$ in DHP was observed at pH 9 in the absence of the azole ligands [415 (Soret), 546, 565 (sh)] and in their presence: Im [417 (Soret), 537, 565 (sh)], Bta [421 (Soret), 546, 577 (sh)], BIm [416 (Soret), 546, 577 (sh)], and Inz [415 (Soret), 546, 577 (sh)] (Figure S2). Qualitatively, the magnitude of the spectral shifts at $\mathrm{pH} 9$ and their interpretation follow the same trends as $\mathrm{pH}$ $7($ Bta $>\operatorname{Im}>$ BIm $>>\operatorname{Inz})$. 
Solution Resonance Raman Studies. Resonance Raman spectra obtained aerobically in 100 $\mathrm{mM} \mathrm{KP}_{\mathrm{i}}(\mathrm{pH} 7)$ containing $10 \% \mathrm{MeOH}(\mathrm{v} / \mathrm{v})$ at $25^{\circ} \mathrm{C}$ are shown in Figure $2 \mathrm{~B}$ for ferric WT DHP B (75 $\mu \mathrm{M}$; black) alone and in the presence of 50 equiv of Im (pink), Bta (blue), BIm (green), and Inz (red), with features reported in Table S2. The DHP core size marker bands $v_{3}$ $\left(1470-1510 \mathrm{~cm}^{-1}\right)$ and $v_{2}\left(1560-1580 \mathrm{~cm}^{-1}\right)$ represent the population distribution of six coordinate vs. five coordinate heme species, respectively, and are sensitive to spin state. ${ }^{42}$ For WT DHP B in the absence of an azole ligand, the presence of $10 \% \mathrm{MeOH}$ in the buffer solution leads to an increase in the 6cHS heme-Fe population (to nearly $100 \%$ ) when compared to aqueous buffer that is normally a mixture of $5 \mathrm{cHS}$ and $6 \mathrm{cHS}$ metaquo heme. ${ }^{43}$ This shift due to the presence of $\mathrm{MeOH}$ has been attributed to a destabilization of the distal histidine (His ${ }^{55}$ ) in the solvent exposed (or "open") conformation in the $10 \% \mathrm{MeOH}$ buffer solution. ${ }^{23}$

The following observations were noted for DHP B in the presence of an azole ligand: imidazole yielded the greatest shift in the DHP heme core size marker bands, from that of a $6 \mathrm{cHS}$ spectrum $\left(v_{3}=1477 \mathrm{~cm}^{-1}, v_{2}=1563 \mathrm{~cm}^{-1}\right.$; black $)$ to one that reflects $6 \mathrm{cLS}\left(v_{3}=1504 \mathrm{~cm}^{-1}\right.$; $v_{2}=1580 \mathrm{~cm}^{-1} ; v_{10}=1643 \mathrm{~cm}^{-1}$; pink). Specifically, the DHP-Im $v_{3}$ LS band was seen at 1504 $\mathrm{cm}^{-1}$ with a small abundance of $v_{3}$ HS at $1471 \mathrm{~cm}^{-1}$, while the $v_{2}$ LS band was noted at $1581 \mathrm{~cm}^{-1}$ with a small shoulder representing the weak abundance of the $v_{2}$ HS band. A slight shoulder at $\sim 1350 \mathrm{~cm}^{-1}$ and an increase at $1617 \mathrm{~cm}^{-1}$ corresponding to the $v_{\mathrm{c}=\mathrm{c}}$ ferrous core size marker band both suggest a minor reduction of DHP occurs in the presence of imidazole that was not observable by UV-visible spectroscopy. With the exception of these ferrous components, the features of DHP-Bta and DHP-BIm were similar to those observed with DHP-Im: core size marker bands were observed at $\sim 1505 \mathrm{~cm}^{-1}$ and $\sim 1581 \mathrm{~cm}^{-1}$, with small scattering for the HS features for both azole adducts. Overall, these three azole ligands yielded spectral features in 
DHP that are consistent with other ferric LS hemoprotein adducts of $\mathrm{DHP}^{44}$, horseradish peroxidase ${ }^{44}$, horse heart myoglobin ${ }^{44}$, and $\mathrm{P}^{4} 50_{\mathrm{BM} 3}{ }^{45-47}$ (Table S2). Finally, while the presence of Im, Bta, and BIm led to a significant shift from a primarily 6cHS heme in DHP to a 6cLS one, DHP-Inz exhibited little to no LS features apart from the typical distribution for WT DHP alone, in agreement with the UV/vis spectroscopic studies (vide supra).

In contrast to the 6cLS heme formed upon azole binding, other DHP substrates lead to a 5cHS ferric heme: the 4-halophenol series, ${ }^{19,}{ }^{48}$ 5-substituted haloindoles, ${ }^{23}$ 4-nitrophenol ${ }^{24}$ and 4-nitrocatechol ${ }^{24}$ all have known binding sites inside the distal pocket that displace the distal $\mathrm{His}^{55}$ to the 'open' conformation (Figure S3A), thereby destabilizing the iron-bound water ligand, and yielding a 5cHS ferric heme. None, however, bind directly to the iron. Thus, the observation of a 6cLS heme for DHP complexes of Im, Bta, and BIm strongly suggests that these three ligands are binding directly to the heme-iron, in agreement with the ligand binding motif revealed by X-ray crystallography (vide infra).

Two additional resonance Raman studies were performed: i) spectra were obtained anaerobically at pH 7 (Figure S4), and showed no significant differences when compared to the aerobic ones; ii) spectra collected aerobically at pH 9 (Figure S5) were similar to those observed at $\mathrm{pH} 7$, with only minor differences that were attributable to the known acid-alkaline transition ${ }^{41}$ in DHP.

Ligand Binding Studies. Using the observed hypochromicity and bathochromic shift of the Soret band upon ligand binding, optical difference spectra were recorded as a function of azole concentration (2.5-100 eq; Figure S6) to determine the apparent dissociation constants $\left(K_{d}\right)$. The $K_{\mathrm{d}}$ values for Im, Bta, and BIm were within $\sim 2$-fold of each other (52-110 $\mu \mathrm{M}$, Table 2). As Inz showed no significant optical changes, no $K_{\mathrm{d}}$ value was determined. When compared to the 
naturally-occurring haloindole peroxygenase substrate 5 -Br-indole $(150 \mu \mathrm{M})$, the three azoles showed higher affinities for DHP, although the comparison is mixed for the other 5-substituted halogenated indoles (5-I-indole: $62 \mu \mathrm{M}$; 5-Cl-indole: $317 \mu \mathrm{M}$ ) that are not native to benthic environments. ${ }^{23}$ The binding affinities for the three azoles were also on a par with, or slightly stronger, than the anthropogenic 4-nitrophenol $(262 \mu \mathrm{M})$, 2,4-dinitrophenol $(105 \mu \mathrm{M})$ and 4nitrocatechol $(40 \mu \mathrm{M})$ substrates, ${ }^{24}$ and were significantly lower than that for the native 4bromophenol $\left(1.15 \mathrm{mM}^{43}\right)$, a known inhibitor ${ }^{49}$ of the enzyme. Additional discussion of the binding affinity of the azoles as informed by X-ray crystallography can be found below.

Table 2. $K_{d}$ Values for Ligand Binding to Ferric DHP B at $\mathrm{pH} 7$.

\begin{tabular}{lll} 
Ligand & $K_{d}(\mu \mathrm{M}), \mathrm{pH} 7$ & Ref. \\
\hline Azole & $52 \pm 2$ & $\mathrm{a}$ \\
$\quad$ imidazole (Im) & $82 \pm 2$ & $\mathrm{a}$ \\
$\quad$ benzotriazole (Bta) & $110 \pm 8$ & $\mathrm{a}$ \\
$\quad$ benzimidazole (BIm) & n. d. ${ }^{\text {b }}$ & $\mathrm{a}$ \\
$\quad$ indazole (Inz) & $62 \pm 10$ & 23 \\
Indole & $150 \pm 10$ & 23 \\
$\quad$ 5-I-indole & $317 \pm 23$ & 23 \\
5-Br-indole & & \\
5-Cl-indole & $40 \pm 1$ & 24 \\
Phenol & $105 \pm 21$ & 24 \\
$\quad$ 4-nitrocatechol & $262 \pm 23$ & 24 \\
2,4-dinitrophenol & 1150 & 43 \\
4-nitrophenol &
\end{tabular}

\section{X-ray Crystallographic Studies of DHP B Complexed with Azoles. Crystal structures} were determined to atomic resolution for all four azole ligands. Data collection and refinement statistics are found in Table S1. Additional single crystal resonance Raman data conducted on crystals prepared in the same manner identified all crystals as being initially in the ferric state, with rapid conversion to the ferrous form following X-ray exposure (vide infra). In each case, 
full occupancy ligand binding was observed in at least one molecule of the DHP B dimer, and the relevant discussion below is thus limited to the results for the best defined ligand sites. All ligands were found in the distal heme pocket, and interacted directly with the iron with the exception of indazole. A comparison of the different binding environments for each ligand is shown in Figure 3, and selected Fe-ligand distances can be found in Table 3.

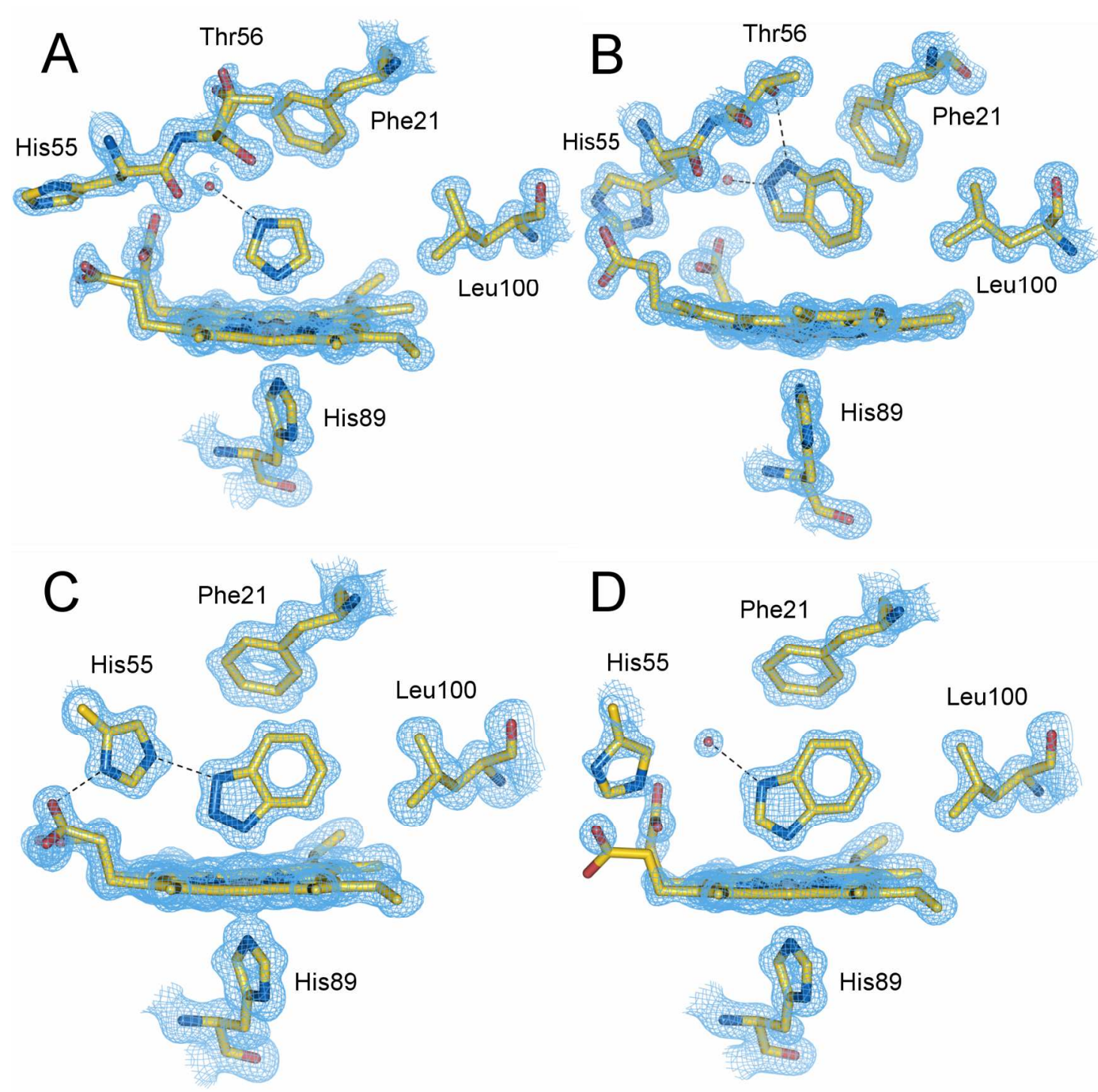

Figure 3. Comparison of the different binding modes at the DHP heme active site by (A) imidazole, (B) indazole, (C) benzotriazole and (D) benzimidazole. Note that in panel (A) only one of two partial occupancy waters that hydrogen bond to imidazole is shown for clarity. 
Table 3: Heme iron-ligand distance for each complex and hydrogen bonds observed.

\begin{tabular}{|c|c|c|c|c|c|c|}
\hline \multirow[b]{2}{*}{ Complex } & \multirow[b]{2}{*}{$\begin{array}{c}\boldsymbol{K}_{\mathbf{d}}(\mu \mathrm{M}) \\
\mathrm{pH} 7^{a}\end{array}$} & \multirow[b]{2}{*}{$\begin{array}{c}\text { Fe-ligand } \\
\text { distance }(\AA)\end{array}$} & \multicolumn{4}{|c|}{ Hydrogen bonds $(\AA)$} \\
\hline & & & $\begin{array}{l}\mathrm{H}_{2} \mathrm{O}- \\
\text { ligand }\end{array}$ & $\begin{array}{c}\mathrm{H}_{2} \mathrm{O}- \\
\text { propionate }\end{array}$ & $\begin{array}{c}\mathrm{H}_{2} \mathrm{O}- \\
\text { Tyr38 }\end{array}$ & $\begin{array}{c}\text { Ligand- } \\
\text { His55 }\end{array}$ \\
\hline Imidazole & $52 \pm 2$ & 2.02 & $2.76 / 2.89^{b}$ & $2.87 / 4.23$ & - & - \\
\hline Benzotriazole & $82 \pm 2$ & 2.05 & - & - & - & 2.65 \\
\hline Benzimidazole & $110 \pm 8$ & 2.05 & 2.78 & 2.88 & - & - \\
\hline Indazole & n. d. ${ }^{c}$ & 3.94 & 2.57 & 2.63 & 2.50 & - \\
\hline
\end{tabular}

For DHP-Im (Figure 3A), the imidazole ligand is observed with full occupancy in both of the active sites of the dimer in the asymmetric unit. It forms a hydrogen bond with a water molecule in the pocket (this water occupies a single position in monomer B, or two alternate partial occupancy water positions in monomer A) and coordinates the iron (Table 3), with His ${ }^{55}$ swung out of the distal pocket. In DHP-Bta (Figure 3C), the benzotriazole ligand interacts directly with the iron at the heme distal pocket, and is stabilized by forming a $2.65 \AA$ hydrogen bond with the $\mathrm{N}^{\varepsilon 2}$ atom of His ${ }^{55}$, which is swung fully into the pocket. Benzotriazole is found in just one of the active sites (monomer B), while in the other one we observe a hemichrome species with His ${ }^{55}$ interacting with the iron and forming a hexacoordinated complex (data not shown). For the DHPBIm adduct (Figure 3D), the benzimidazole ligand also complexes the heme-iron in the axial position resulting in a hexacoordinate species, and forms a hydrogen bond with a water present in the pocket. Benzimidazole is found in both active sites with good occupancy. For all the double ring aromatic ligands, residues Phe 21, Phe 35, Val 59 and Leu 100 provide a hydrophobic environment in the pocket to favour their binding. A superposition of the DHP-Im, DHP-BIm and DHP-Bta binding modes, together with the positioning of indazole in the pocket, is given in Figure 4. The ligands superimpose well, albeit with the smaller Im being rotated slightly away from $\mathrm{His}^{55}$. The residues forming the hydrophobic pocket Phe24, Phe35, Val59, 
Leu100, are in near-identical orientations in all three structures. Notably, for Bta and BIm, Phe21 is forced to rotate $\sim 13^{\circ}$ away from the heme in order to accommodate the larger ligand. A shift in the protein backbone occurs in the DHP-Bta structure, likely the result of the hydrogen bond formation between His55 and the bound ligand.

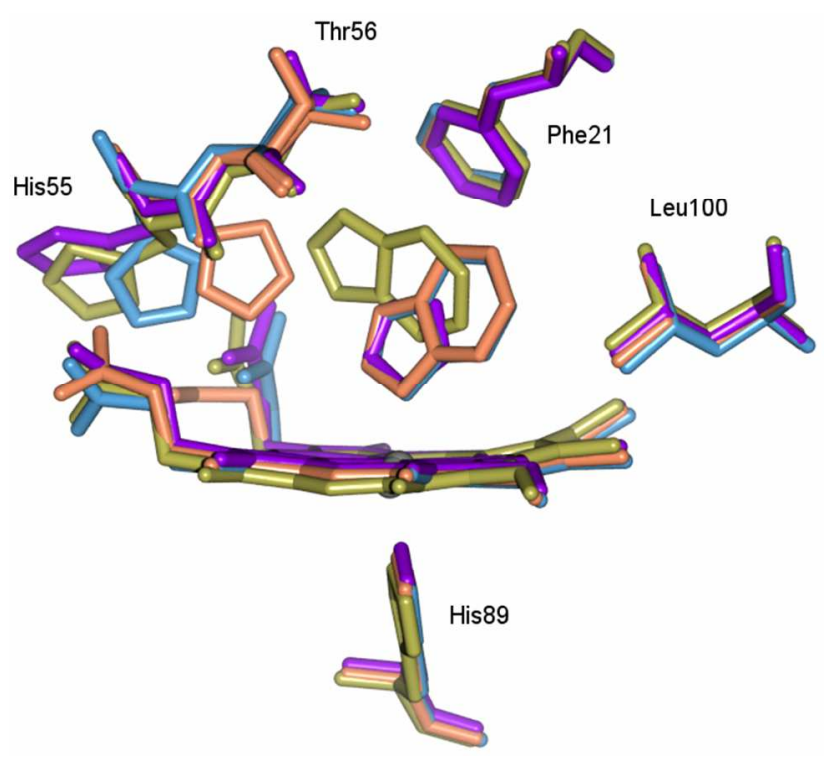

Figure 4. Superposition of the active site for the azole-DHP complexes, showing the binding environment with the most relevant residues in each case. Imidazole is shown in purple, indazole in gold, benzimidazole in blue, and benzotriazole in orange.

In contrast, in the case of DHP-Inz (Figure 3B), the indazole ligand is found with full occupancy in the distal pocket, but does not complex the heme-iron. Rather, it is located more distant from the heme plane than the other ligands, and presents hydrogen bond interactions with $\mathrm{Thr}^{56}-\mathrm{O}^{\gamma 1}$ and a neighbouring water molecule. It also has a hydrophobic stacking interaction with the aromatic ring of Phe 21 , with the ring planes close to parallel and separated by $\sim 4 \AA$. Overall, the ligand binding motif in DHP-Inz is in good agreement with the observations noted above for both UV-visible and resonance Raman spectroscopies that suggested a HS heme persists in DHP even in the presence of the indazole ligand.

Single crystal resonance Raman data were measured from separate DHP-azole crystals 
(Figure S7 and Table S3), grown and soaked in the same manner as described above, in order to facilitate comparison with solution data and to monitor redox and ligand states. The 5c ferric heme in DHP-Inz crystals was highly susceptible to photoreduction by the $405 \mathrm{~nm}$ excitation laser, while the $6 \mathrm{c}$ hemes of the remaining complexes were not, provided that relatively low laser powers were used. All crystals underwent rapid reduction in the X-ray beam consistent with previous reports on cryo-radiolytic reduction of heme proteins. ${ }^{50}$ This suggests that the DHPazole structures represent a mixture of ferric and (predominantly) ferrous hemes.

Computational Studies. To investigate azole binding and the possible ligation with the heme Fe, a density functional theory (DFT) computational approach was utilized. All structures converged to the binding of the free azole nitrogen to the iron, as seen in Figure S8 and Table S4. For Bta, there are two possible binding conformations (Figure S8D and E) that are $<1 \mathrm{kcal} / \mathrm{mol}$ different in energy, however the binding through the N2 nitrogen is stronger (Fe-N distance of $2.038 \AA$ ) versus $2.087 \AA$ through N3. The crystal structure shows only one binding conformation for Bta (Figure 3C) that matches closely to the calculated conformation in Figure S8D. The imidazole DFT structure showed coordination to the iron through N2 with a Fe-N distance of $2.04 \AA$ (Figure S8A), which matches nicely with the crystal structure (Figure 3A). BIm also showed heme ligation through BIm N2 with an Fe-N distance of $2.040 \AA$ (Figure S8B) that matches well with the observed crystal structure (Figure 3D). Indazole was calculated to bind in a similar fashion to the Bta conformation (Figure S8C and E, respectively), but in the crystal structure is shown to bind in the distal pocket $\sim 4.0 \AA$ away and not directly coordinate to the heme iron (Figure 3B). Bta (E) and Inz (C) likely do not bind in these conformations due to steric effects with other residues inside the distal pocket (Figure S9, see Discussion below).

Biochemical Assays. The azoles were explored as possible peroxidase/peroxygenase 
substrates for DHP (see Supporting Information for experimental details). However, HPLC analysis revealed that under all conditions tested (variable $\mathrm{pH}$, variable $\mathrm{H}_{2} \mathrm{O}_{2}$ concentration), no oxidation nor degradation of these compounds was noted (data not shown). Further, stoppedflow UV-visible spectroscopic studies of the two high-valent iron-oxo species typically invoked in hemoprotein reactions, Compounds $\mathrm{I}^{29}$ and $\mathrm{ES}^{27}$, when pre-formed and reacted with 10-100 eq. of azole, showed that neither were able to oxidize the azoles (data not shown).

Given their $K_{\mathrm{d}}$ values of $\sim 50-110 \mu \mathrm{M}$, the azoles were explored as possible inhibitors of peroxygenase activity: $10 \mu \mathrm{M}$ ferric WT DHP B was preincubated with 50-750 mol equivalents azole, reacted with 50 eq. each of 5-bromoindole and $\mathrm{H}_{2} \mathrm{O}_{2}$, and then subjected to HPLC analysis. Substrate conversion of 5-bromoindole was uninhibited over the azole concentration range analyzed. This differs from the known inhibitor ${ }^{49}$ 4-bromophenol $\left(K_{\mathrm{d}}=1.15 \mathrm{mM}^{43}\right)$ that inhibits both peroxygenase and peroxidase activities. We rationalize this counterintuitive result by the following: the weaker binding 4-bromophenol inhibitor binds internally in the distal pocket (without directly ligating the heme iron), thereby inhibiting substrate binding. On the other hand, although Im, Bta, and BIm directly coordinate to the iron, the binding of $\mathrm{H}_{2} \mathrm{O}_{2}$ is stronger: we estimate the $K_{\mathrm{d}}$ of $\mathrm{H}_{2} \mathrm{O}_{2}$ to have an upper bound equal to the $K_{M}^{H 2 O 2}$ value of $35 \mu \mathrm{M}$ (from Michaelis-Menten kinetics: if $\mathrm{k}_{1}<<\mathrm{k}_{-1}$, then $K_{\mathrm{M}}=K_{\mathrm{d}}$; if $\mathrm{k}_{1} \geq \mathrm{k}_{-1}$, then $K_{\mathrm{M}}>K_{\mathrm{d}}$ ). Therefore, $\mathrm{H}_{2} \mathrm{O}_{2}$ is able to displace the coordinated azole ligands, allowing for both enzyme activation and substrate turnover.

\section{DISCUSSION}

Given its native peroxygenase activity against naturally-occurring halogenated indoles, the aim of the present study was to explore if DHP is able to bind, and/or potentially react with, 
structurally related compounds of anthropogenic origin. Typically, inhibitors of $\mathrm{O}_{2}$ transport in hemoglobins are small molecules (e.g. carbon monoxide, cyanide, azide, nitric oxide) owing to the lack of an active site pocket capable of allowing access to facilitate binding of larger ligands. ${ }^{51}$ As such, larger N-containing heterocyclic compounds, such as those found in the azoles investigated here, would not be considered as ligands capable of binding in a globin active site, let alone coordinate to the heme-iron. However, owing to its multifunctional nature as a detoxification enzyme that is unrelated to its primary function as an $\mathrm{O}_{2}$ transport globin, DHP possesses an active site pocket capable of halophenol and haloindole binding that supports both peroxidase and peroxygenase activities, respectively. ${ }^{25}$

We have previously postulated that when one considers the known substrate binding sites for halophenols, haloindoles, and nitrophenols in DHP, there is evidence to suggest at least two different substrate binding motifs (Figure S3B). ${ }^{25}$ The first binding motif is observed for the peroxidase substrates 2,4,6-tribromophenol (TBP) and 2,4,6-trichlorophenol (TCP): both TBP (in WT DHP A $)^{52}$ and TCP [in the DHP A(Y34N) mutant $]^{53}$ deeply bind in the distal cavity near the $\alpha$ edge of the heme in close proximity to L100, the substrates (but not the bromine atoms) encompass the $\mathrm{Xe}$ binding site, the phenolic-OH is pointed towards the heme $\mathrm{Fe}$, and the substrate is far enough removed that His ${ }^{55}$ is found in both the 'open' and the 'closed' (internal) conformations. ${ }^{42,43}$ The second motif is observed for 4-nitrophenol, 4-nitrocatetchol, and the 4halophenol series: these substrates bind above the heme with a tilt away from the $\beta$ edge, the aromatic rings are oriented for $\pi$-stacking interactions with F21, the halogen and nitro substituents of the phenolic substrates are positioned internally in the Xe1 binding site, the phenolic-OH is situated near the pocket entrance with stabilizing $\mathrm{H}$-bonding interactions to the heme propionate D and/or Y38, and the distal histidine (His55) is swung into the solvent exposed 
'open' conformation. ${ }^{24}$ TCP has also been shown to bind in an alternate orientation in the DHP $\mathrm{A}(\mathrm{Y} 34 \mathrm{~N} / \mathrm{S} 91 \mathrm{G})$ double mutant that more closely resembles this second binding motif: situated at the heme $\gamma$ edge between both heme propionate arms, the phenolic-OH hydrogen bonds to Y38, the $\mathrm{Cl}$ substituent of the C4 carbon points toward the heme-Fe, and His ${ }^{55}$ is found in the 'open' conformation. $^{53}$

In the case of the azole compounds Bta, BIm, and Im, a third ligand binding motif is now identified, one in which the ligand binds directly to the heme-Fe through a coordinate covalent bond from a nitrogen atom, resulting in a hexacoordinate low spin (6cLS) adduct. Such a direct ligation of N-heterocyclic compounds to heme proteins has been previously studied in the cytochrome P450 monooxygenases: organic building blocks such as imidazoles and triazoles, among other azole derivatives found in pharmaceuticals, have been shown to coordinate directly to heme iron through the nitrogen, termed type II binding ligands. ${ }^{54}$ The binding of azole compounds to P450s has been shown to be inhibitory owing to the change in spin and coordination state from a $5 \mathrm{cHS}$ to a $6 \mathrm{cLS}$ heme, thus making it more difficult for reduction to take place to initiate the P450 catalytic cycle. ${ }^{54,55}$ In the case of DHP, the azole derivatives that were explored in this work also lead to this spin state shift upon their binding, resulting in significant optical changes similar to those observed with the P450s. However, unlike the inhibition that is seen with the P450s in the presence of azole compounds, there is no hindrance on the peroxidase and peroxygenase activities of DHP as such an initial reduction is not invoked in the DHP peroxidase/peroxygenase catalytic cycles. Rather, binding of $\mathrm{H}_{2} \mathrm{O}_{2}$ likely displaces the azole ligands, leading to enzyme activation via the resulting reactive ferryl species.

The DFT computational results, performed in the absence of the protein using a model heme, support a direct ligation of all four azole compounds to the heme iron. In the case of imidazole 
and benzimidazole, the calculated binding through the N3 nitrogen is in good agreement with that observed by X-ray crystallography. For benzotriazole, two different binding geometries of nearly degenerate energy were calculated, one through the N2 nitrogen, the other through N3. However, the crystallographically observed ligand binding geometry matches that of the N2 conformation, likely due to steric hindrance with the protein that occurs with the more 'vertical' orientation of the azole ligand (Figure S9). Interestingly, indazole was also calculated to bind through the N2 nitrogen in this 'vertical' arrangement owing to protonation at N1 (the 1-H tautomer of indazole is $15.1 \mathrm{~kJ} / \mathrm{mol}$ more stable than the $2-\mathrm{H}$ tautomer ${ }^{56}$ ), but was found to not bind directly through either nitrogen by X-ray crystallography; rather, indazole binding in the hydrophobic pocket more closely resembles the second motif described above for 4-nitrophenol, 4-nitrocatetchol, and the 4-halophenol series. We surmise that the observed binding orientation of indazole in the DHP active site pocket must be more favorable than the annular tautomerization needed for N1 binding and direct ligation to the heme iron, with steric hindrance precluding the 'vertical' binding arrangement required for N2 binding (Figure S9).

Consistent with the postulate that small-molecule binding sites may exist with regulatory implications in DHP, we have put forth that both the conformational flexibility ('open' vs. 'closed', Figure S3A) and the tautomeric state of the distal histidine, His ${ }^{55}$, may govern how DHP switches between its hemoglobin and enzymatic activities upon substrate binding. ${ }^{48,}$ 57-60 Although the azoles themselves are not substrates, this conformational flexibility of the distal histidine is well-highlighted from the distances between Fe and $\mathrm{His}^{55}$ of the four azole-bound structures of DHP, from the fully 'open' conformation of DHP-Im $(9.47 \AA)$, to the progressively

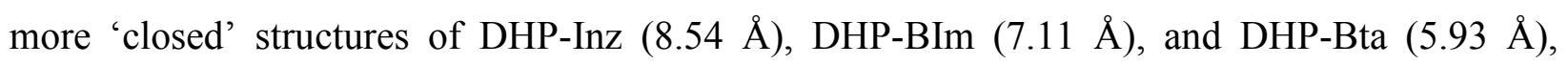
Figure 4 (note: the shorter of the $\mathrm{Fe}-\mathrm{N}^{\varepsilon 2}$ or $\mathrm{Fe}-\mathrm{N}^{\delta 1}$ distances is given, or in the case of DHP-BIm, 
$\mathrm{Fe}-\mathrm{C}^{\delta 2}$ ). The movement of His55 encompasses a side chain reorientation but also a shift of the protein backbone (Figure S3C). Thus, the potential physiological consequences of azole binding to the DHP hemoglobin are two-fold: first, being directly bound to the heme-Fe, the azole could possibly sterically hinder $\mathrm{O}_{2}$-binding to a ferrous heme, and second, the distal histidine would need to undergo substantial active site reorganization to effectively stabilize an oxyferrous adduct. This is particularly noteworthy, as DHP already exhibits a much lower $\mathrm{O}_{2}$ affinity $\left(K_{\mathrm{O} 2}=\right.$ $3.23 \mu \mathrm{M})$ than observed for sperm whale myoglobin $\left(K_{\mathrm{O} 2}=0.27 \mu \mathrm{M}\right)$, a direct consequence that arises from the need for the distal histidine in DHP, with a $\mathrm{N}^{\alpha 2}-\mathrm{Fe}$ distance of $5.4 \AA$, to serve two roles: dioxygen stabilization and acid/base catalyst. ${ }^{61,} 62$ By comparison, in globins the corresponding distance is 4.1-4.6 $\AA^{63}$ [e.g., human $\mathrm{Hb}: 4.24 \AA\left(2 \mathrm{~W} 6 \mathrm{~V}^{64}\right)$; equine hemoglobin: $4.35 \AA\left(1 \mathrm{NS} 9^{65}\right)$; sperm whale $\left.\mathrm{Mb}: 4.61 \AA\left(1 \mathrm{BZP}^{66}\right)\right]$, where it is well positioned to stabilize the oxyferrous adduct formed upon $\mathrm{O}_{2}$-binding to the ferrous heme. For peroxidases, the distance is greater $(5.5-6.0 \AA)$, where the role of the distal histidine is to serve as the general acid/base catalyst that facilitates $\mathrm{H}_{2} \mathrm{O}_{2}$ heterolysis, leading to enzyme activation. Thus, based on the results from the present study, we suggest that azole binding has the potential to disrupt hemoglobin function, although the effects of this possibility still need to be investigated in more detail.

The UV-visible and resonance Raman spectroscopic data are in excellent agreement with the structural characterization of azole binding afforded by X-ray crystallography, and are well supported by our computational work. Together, they provide strong evidence for the direct binding of the azole ligand to the heme iron, leading to a hexacoordinate low-spin adduct. Although not the direct subject of the present study, the physiological implications for marine annelid survival are significant: taken together, the results suggest a new hypothesis where azole pollutants can potentially disrupt hemoglobin function, and on this basis suggest a much larger 
number of structurally related compounds may need to be re-evaluated as potential marine environmental contaminants that pose significant health risks for aquatic organisms. Future studies will focus on the evaluating the oxygen binding affinity of dehaloperoxidase as a function of azole ligand, to further elucidate if this newly identified interaction represents a previously unknown mode of toxicity.

\title{
ASSOCIATED CONTENT \\ Supporting Information. Optical spectra, titration curves, and resonance Raman spectra (solution and single crystal) of DHP-azole complexes, data and refinement statistics for DHP B ferric crystals complexed with azole ligands, and geometry optimized structures. This material is available free of charge via the Internet at http://pubs.acs.org.
}

\author{
AUTHOR INFORMATION \\ Corresponding Authors \\ *Email: Reza_Ghiladi@ncsu.edu; Phone: 1-919-513-0680 \\ *Email: mahough@essex.ac.uk; Phone: 441206873317
}

\section{Notes}

The authors declare no competing financial interest.

\section{ACKNOWLEDGMENT}

This project was supported by NSF CAREER Award CHE-1150709 (R.G.), NSF CHE-1609446 (S.F. and R.G.), and Leverhulme Trust Project Grant RPG-2014-355 (T.M.C. and M.H.). We 
thank Dr. Florian Dworkowski of the Paul Scherrer Institut/SLS for assistance with SCRR measurements.

\section{REFERENCES}

1. Giger, W., Schaffner, C., and Kohler, H.-P. E. (2006) Benzotriazole and Tolyltriazole as Aquatic Contaminants. 1. Input and Occurrence in Rivers and Lakes, Environ. Sci. Technol. 40, 7186-7192.

2. Cancilla, D. A., Martinez, J., and van Aggelen, G. C. (1998) Detection of Aircraft Deicing/Antiicing Fluid Additives in a Perched Water Monitoring Well at an International Airport, Environ. Sci. Technol. 32, 3834-3835.

3. Weiss, S., Jakobs, J., and Reemtsma, T. (2006) Discharge of Three Benzotriazole Corrosion Inhibitors with Municipal Wastewater and Improvements by Membrane Bioreactor Treatment and Ozonation, Environ. Sci. Technol. 40, 7193-7199.

4. Reemtsma, T., Miehe, U., Duennbier, U., and Jekel, M. (2010) Polar pollutants in municipal wastewater and the water cycle: Occurrence and removal of benzotriazoles, Water Res. 44, 596-604.

5. Loos, R., Locoro, G., Comero, S., Contini, S., Schwesig, D., Werres, F., Balsaa, P., Gans, O., Weiss, S., Blaha, L., Bolchi, M., and Gawlik, B. M. (2010) Pan-European survey on the occurrence of selected polar organic persistent pollutants in ground water, Water Res. 44, 4115-4126.

6. Rodríguez, J. J. S., Padrón, M. E. T., Aufartová, J., and Ferrera, Z. S. (2010) Benzimidazole Fungicides in Environmental Samples: Extraction and Determination Procedures, In Fungicides (Carisse, O., Ed.), InTech.

7. Carlile, B. (2006) Pesticide Selectivity, Health and the Environment, Cambridge University Press.

8. Hof, H. (2001) Critical Annotations to the Use of Azole Antifungals for Plant Protection, Antimicrob. Agents Chemother. 45, 2987-2990.

9. Oh, S. J., Park, J., Lee, M. J., Park, S. Y., Lee, J.-H., and Choi, K. (2006) Ecological hazard assessment of major veterinary benzimidazoles: Acute and chronic toxicities to aquatic microbes and invertebrates, Environ. Toxicol. Chem. 25, 2221-2226.

10. Beynon, S. A. (2012) Potential environmental consequences of administration of anthelmintics to sheep, Vet. Parasitol. 189, 113-124.

11. Gaikwad, D. D., Chapolikar, A. D., Devkate, C. G., Warad, K. D., Tayade, A. P., Pawar, R. P., and Domb, A. J. (2015) Synthesis of indazole motifs and their medicinal importance: An overview, Eur. J. Med. Chem. 90, 707-731.

12. Vandeputte, P., Ferrari, S., and Coste, A. T. (2012) Antifungal Resistance and New Strategies to Control Fungal Infections, Int. J. Microbiol. 2012, 26 pages, Article ID 713687

13. Andersson Trojer, M., Movahedi, A., Blanck, H., and Nyden, M. (2013) Imidazole and Triazole Coordination Chemistry for Antifouling Coatings, J. Chem. 2013, 23 pages, Article ID 946739. 
14. Forte, B., Malgesini, B., Piutti, C., Quartieri, F., Scolaro, A., and Papeo, G. (2009) A Submarine Journey: The Pyrrole-Imidazole Alkaloids, Marine Drugs 7, 705-753.

15. Reddy, C. M., Quinn, J. G., and King, J. W. (2000) Free and Bound Benzotriazoles in Marine and Freshwater Sediments, Environ. Sci. Technol. 34, 973-979.

16. Muirhead-Thomson, R. C. (2009) Pesticide Impact on Stream Fauna: With Special Reference to Macroinvertebrates, Cambridge University Press, Cambridge.

17. Weber, R. E., Mangum, C., Steinman, H., Bonaventura, C., Sullivan, B., and Bonaventura, J. (1977) Hemoglobins of two terebellid polychaetes: Enoplobranchus sanguineus and Amphitrite ornata, Comp. Biochem. Physiol. A Comp. Physiol. 56, 179187.

18. Franzen, S., Thompson, M. K., and Ghiladi, R. A. (2012) The dehaloperoxidase paradox, Biochim. Biophys. Acta 1824, 578-588.

19. Lebioda, L., LaCount, M. W., Zhang, E., Chen, Y. P., Han, K., Whitton, M. M., Lincoln, D. E., and Woodin, S. A. (1999) An enzymatic globin from a marine worm, Nature 401, 445.

20. Franzen, S., Sasan, K., Sturgeon, B. E., Lyon, B. J., Battenburg, B. J., Gracz, H., Dumariah, R., and Ghiladi, R. (2012) Nonphotochemical base-catalyzed hydroxylation of 2,6-dichloroquinone by $\mathrm{H}_{2} \mathrm{O}_{2}$ occurs by a radical mechanism, J. Phys. Chem. B 116, 1666-1676.

21. D'Antonio, E. L., D'Antonio, J., de Serrano, V., Gracz, H., Thompson, M. K., Ghiladi, R. A., Bowden, E. F., and Franzen, S. (2011) Functional consequences of the creation of an Asp-His-Fe triad in a 3/3 globin, Biochemistry 50, 9664-9680.

22. Han, K., Woodin, S. A., Lincoln, D. E., Fielman, K. T., and Ely, B. (2001) Amphitrite ornata, a marine worm, contains two dehaloperoxidase genes, Mar. Biotechnol. (NY) 3, 287-292.

23. Barrios, D. A., D'Antonio, J., McCombs, N. L., Zhao, J., Franzen, S., Schmidt, A. C., Sombers, L. A., and Ghiladi, R. A. (2014) Peroxygenase and oxidase activities of dehaloperoxidase-hemoglobin from Amphitrite ornata, J. Am. Chem. Soc. 136, 79147925.

24. McCombs, N. L., D’Antonio, J., Barrios, D. A., Carey, L. M., and Ghiladi, R. A. (2016) Nonmicrobial Nitrophenol Degradation via Peroxygenase Activity of DehaloperoxidaseHemoglobin from Amphitrite ornata, Biochemistry 55, 2465-2478.

25. Franzen, S., Ghiladi, R. A., Lebioda, L., and Dawson, J. (2016) Chapter 10 Multifunctional Hemoglobin Dehaloperoxidases, In Heme Peroxidases, pp 218-244, The Royal Society of Chemistry.

26. Beers, R. F., Jr., and Sizer, I. W. (1952) A spectrophotometric method for measuring the breakdown of hydrogen peroxide by catalase, J. Biol. Chem. 195, 133-140.

27. Feducia, J., Dumarieh, R., Gilvey, L. B., Smirnova, T., Franzen, S., and Ghiladi, R. A. (2009) Characterization of dehaloperoxidase compound ES and its reactivity with trihalophenols, Biochemistry 48, 995-1005.

28. D'Antonio, J., D'Antonio, E. L., Thompson, M. K., Bowden, E. F., Franzen, S., Smirnova, T., and Ghiladi, R. A. (2010) Spectroscopic and mechanistic investigations of dehaloperoxidase B from Amphitrite ornata, Biochemistry 49, 6600-6616.

29. Dumarieh, R., D'Antonio, J., Deliz-Liang, A., Smirnova, T., Svistunenko, D. A., and Ghiladi, R. A. (2013) Tyrosyl Radicals in Dehaloperoxidase: How nature deals with 
evolving an oxygen-binding globin to a biologically relevant peroxidase, J. Biol. Chem. 288, 33470-33482.

30. Chenprakhon, P., Sucharitakul, J., Panijpan, B., and Chaiyen, P. (2010) Measuring Binding Affinity of Protein-Ligand Interaction Using Spectrophotometry: Binding of Neutral Red to Riboflavin-Binding Protein, J. Chem. Educ. 87, 829-831.

31. Pompidor, G., Dworkowski, F. S. N., Thominet, V., Schulze-Briese, C., and Fuchs, M. R. (2013) A new on-axis micro-spectrophotometer for combining Raman, fluorescence and UV/Vis absorption spectroscopy with macromolecular crystallography at the Swiss Light Source, J. Synchrotron Radiat. 20, 765-776.

32. Dworkowski, F. S. N., Hough, M. A., Pompidor, G., and Fuchs, M. R. (2015) Challenges and solutions for the analysis of in situ, in crystallo micro-spectrophotometric data, Acta Crystallographica Section D: Biological Crystallography 71, 27-35.

33. Kabsch, W. (2010) XDS, Acta Crystallogr D Biol Crystallogr 66, 125-132.

34. Evans, P. R., and Murshudov, G. N. (2013) How good are my data and what is the resolution?, Acta Crystallogr D Biol Crystallogr 69, 1204-1214.

35. Kleywegt, G. J. (2007) Crystallographic refinement of ligand complexes, Acta Crystallogr D Biol Crystallogr 63, 94-100.

36. Chen, V. B., Arendall, W. B., 3rd, Headd, J. J., Keedy, D. A., Immormino, R. M., Kapral, G. J., Murray, L. W., Richardson, J. S., and Richardson, D. C. (2010) MolProbity: allatom structure validation for macromolecular crystallography, Acta Crystallogr D Biol Crystallogr 66, 12-21.

37. Zeldin, O. B., Gerstel, M., and Garman, E. F. (2013) RADDOSE-3D: time- and spaceresolved modelling of dose in macromolecular crystallography, J. Appl. Crystallogr. 46, 1225-1230.

38. Schäfer, A., Horn, H., and Ahlrichs, R. (1992) Fully optimized contracted Gaussian basis sets for atoms Li to Kr, J. Chem. Phys. 97, 2571-2577.

39. Ghiladi, R. A., Medzihradszky, K. F., Rusnak, F. M., and Ortiz de Montellano, P. R. (2005) Correlation between isoniazid resistance and superoxide reactivity in Mycobacterium tuberculosis KatG, J. Am. Chem. Soc. 127, 13428-13442.

40. Ghiladi, R. A., Medzihradszky, K. F., and Ortiz de Montellano, P. R. (2005) The role of the Met-Tyr-Trp crosslink in Mycobacterium tuberculosis catalase-peroxidase (KatG) as revealed by KatG(M255I), Biochemistry 44, 15093-15105.

41. Nienhaus, K., Deng, P., Belyea, J., Franzen, S., and Nienhaus, G. U. (2006) Spectroscopic study of substrate binding to the carbonmonoxy form of dehaloperoxidase from Amphitrite ornata, J. Phys. Chem. B 110, 13264-13276.

42. Nicoletti, F. P., Thompson, M. K., Howes, B. D., Franzen, S., and Smulevich, G. (2010) New insights into the role of distal histidine flexibility in ligand stabilization of dehaloperoxidase-hemoglobin from Amphitrite ornata, Biochemistry 49, 1903-1912.

43. Thompson, M. K., Davis, M. F., de Serrano, V., Nicoletti, F. P., Howes, B. D., Smulevich, G., and Franzen, S. (2010) Internal binding of halogenated phenols in dehaloperoxidase-hemoglobin inhibits peroxidase function, Biophys. J. 99, 1586-1595.

44. Belyea, J., Belyea, C. M., Lappi, S., and Franzen, S. (2006) Resonance Raman study of ferric heme adducts of dehaloperoxidase from Amphitrite ornata, Biochemistry 45, $14275-14284$. 
45. Deng, T. J., Proniewicz, L. M., Kincaid, J. R., Yeom, H., Macdonald, I. D., and Sligar, S. G. (1999) Resonance Raman studies of cytochrome P450BM3 and its complexes with exogenous ligands, Biochemistry 38, 13699-13706.

46. Smith, S. J., Munro, A. W., and Smith, W. E. (2003) Resonance Raman scattering of cytochrome P450 BM3 and effect of imidazole inhibitors, Biopolymers 70, 620-627.

47. Macdonald, I. D., Smith, W. E., and Munro, A. W. (1996) Inhibitor/fatty acid interactions with cytochrome P-450 BM3, FEBS Lett. 396, 196-200.

48. Davis, M. F., Gracz, H., Vendeix, F. A., de Serrano, V., Somasundaram, A., Decatur, S. M., and Franzen, S. (2009) Different modes of binding of mono-, di-, and trihalogenated phenols to the hemoglobin dehaloperoxidase from Amphitrite ornata, Biochemistry 48, 2164-2172.

49. Zhao, J., and Franzen, S. (2013) Kinetic study of the inhibition mechanism of dehaloperoxidase-hemoglobin a by 4-bromophenol, J. Phys. Chem. B 117, 8301-8309.

50. Beitlich, T., Kuhnel, K., Schulze-Briese, C., Shoeman, R. L., and Schlichting, I. (2007) Cryoradiolytic reduction of crystalline heme proteins: analysis by UV-Vis spectroscopy and X-ray crystallography, J. Synchrotron Radiat. 14, 11-23.

51. Perutz, M. F. (1990) Mechanisms regulating the reactions of human hemoglobin with oxygen and carbon monoxide, Annu. Rev. Physiol. 52, 1-25.

52. Zhao, J., de Serrano, V., Le, P., and Franzen, S. (2013) Structural and kinetic study of an internal substrate binding site in dehaloperoxidase-hemoglobin A from Amphitrite ornata, Biochemistry 52, 2427-2439.

53. Wang, C., Lovelace, L. L., Sun, S., Dawson, J. H., and Lebioda, L. (2013) Complexes of dual-function hemoglobin/dehaloperoxidase with substrate 2,4,6-trichlorophenol are inhibitory and indicate binding of halophenol to compound I, Biochemistry 52, 62036210 .

54. Locuson, C. W., Hutzler, J. M., and Tracy, T. S. (2007) Visible Spectra of Type II Cytochrome P450-Drug Complexes: Evidence that "Incomplete" Heme Coordination Is Common, Drug Metab. Dispos. 35, 614-622.

55. Hutzler, J. M., Melton, R. J., Rumsey, J. M., Schnute, M. E., Locuson, C. W., and Wienkers, L. C. (2006) Inhibition of Cytochrome P450 3A4 by a Pyrimidineimidazole: Evidence for Complex Heme Interactions, Chem. Res. Toxicol. 19, 1650-1659.

56. Alkorta, I., and Elguero, J. (2005) Theoretical estimation of the annular tautomerism of indazoles, J. Phys. Org. Chem. 18, 719-724.

57. Chen, Z., de Serrano, V., Betts, L., and Franzen, S. (2009) Distal histidine conformational flexibility in dehaloperoxidase from Amphitrite ornata, Acta Cryst. D65, 34-40.

58. Smirnova, T. I., Weber, R. T., Davis, M. F., and Franzen, S. (2008) Substrate binding triggers a switch in the iron coordination in dehaloperoxidase from Amphitrite ornata: HYSCORE experiments, J. Am. Chem. Soc. 130, 2128-2129.

59. de Serrano, V., D'Antonio, J., Franzen, S., and Ghiladi, R. A. (2010) Structure of dehaloperoxidase $\mathrm{B}$ at $1.58 \mathrm{~A}$ resolution and structural characterization of the $\mathrm{AB}$ dimer from Amphitrite ornata, Acta Cryst. D66, 529-538.

60. Zhao, J., de Serrano, V., Dumarieh, R., Thompson, M., Ghiladi, R. A., and Franzen, S. (2012) The role of the distal histidine in $\mathrm{H}_{2} \mathrm{O}_{2}$ activation and heme protection in both peroxidase and globin functions, J. Phys. Chem. B 116, 12065-12077.

61. Du, J., Huang, X., Sun, S., Wang, C., Lebioda, L., and Dawson, J. H. (2011) Amphitrite ornata Dehaloperoxidase (DHP): Investigations of Structural Factors That Influence the 
Mechanism of Halophenol Dehalogenation Using "Peroxidase-like" Myoglobin Mutants and "Myoglobin-like" DHP Mutants, Biochemistry 50, 8172-8180.

62. Sun, S., Sono, M., Wang, C., Du, J., Lebioda, L., and Dawson, J. H. (2014) Influence of heme environment structure on dioxygen affinity for the dual function Amphitrite ornata hemoglobin/dehaloperoxidase. Insights into the evolutional structure-function adaptations, Arch. Biochem. Biophys. 545, 108-115.

63. Matsui, T., Ozaki, S., Liong, E., Phillips, G. N., Jr., and Watanabe, Y. (1999) Effects of the location of distal histidine in the reaction of myoglobin with hydrogen peroxide, $J$. Biol. Chem. 274, 2838-2844.

64. Savino, C., Miele, A. E., Draghi, F., Johnson, K. A., Sciara, G., Brunori, M., and Vallone, B. (2009) Pattern of cavities in globins: The case of human hemoglobin, Biopolymers 91, 1097-1107.

65. Robinson, V. L., Smith, B. B., and Arnone, A. (2003) A pH-Dependent Aquomet-toHemichrome Transition in Crystalline Horse Methemoglobin, Biochemistry 42, 1011310125.

66. Kachalova, G. S., Popov, A. N., and Bartunik, H. D. (1999) A Steric Mechanism for Inhibition of CO Binding to Heme Proteins, Science 284, 473-476.

TOC Graphic (For Table of Contents Only)

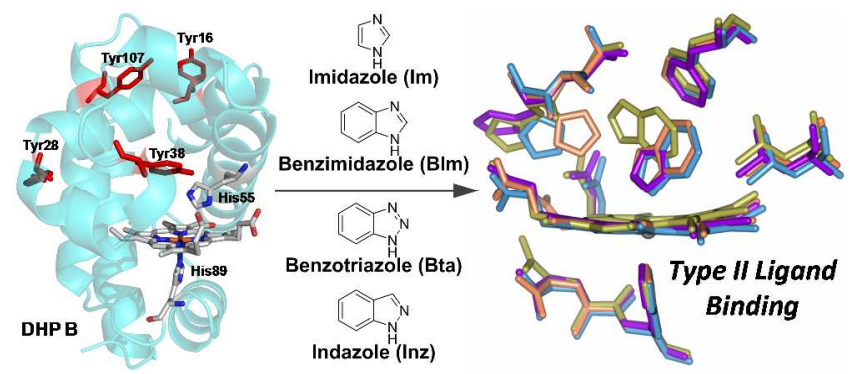

